\title{
Long-Term Depression in the Adult Hippocampus In Vivo Involves Activation of Extracellular Signal-Regulated Kinase and Phosphorylation of Elk-1
}

\author{
Edda Thiels, ${ }^{1,2}$ Beatriz I. Kanterewicz, ${ }^{1}$ Eric D. Norman, ${ }^{1,2}$ James M. Trzaskos, ${ }^{3}$ and Eric Klann ${ }^{1,2}$ \\ ${ }^{1}$ Department of Neuroscience and ${ }^{2}$ Center for the Neural Basis of Cognition, University of Pittsburgh, Pittsburgh, \\ Pennsylvania 15260, and ${ }^{3}$ DuPont Pharmaceuticals Research Laboratory, Wilmington, Delaware 19880
}

Protein kinase cascades likely play a critical role in the signaling events that underlie synaptic plasticity and memory. The extracellular signal-regulated kinase (ERK) cascade is suited well for such a role because its targets include regulators of gene expression. Here we report that the ERK cascade is recruited during long-term depression (LTD) of synaptic strength in area CA1 of the adult hippocampus in vivo and selectively impacts on phosphorylation of the nuclear transcription factor Elk-1. Using a combination of in vivo electrophysiology, biochemistry, pharmacology, and immunohistochemistry, we found the following: (1) ERK phosphorylation, including phosphorylation of nuclear ERK, and ERK phosphotransferase activity are increased markedly, albeit transiently, after the induction of NMDA receptor-dependent LTD at the commissural input to area CA1 pyramidal cells in the hippocampus of anesthetized adult rats; (2) LTD-inducing paired-pulse stimulation fails to produce lasting LTD in the presence of the ERK kinase inhibitor SL327, which suggests that ERK activation is necessary for the persistence of LTD; and (3) ERK activation during LTD results in increased phosphorylation of Elk-1 but not of the transcription factor cAMP response element-binding protein. Our findings indicate that the ERK cascade transduces signals from the synapse to the nucleus during LTD in hippocampal area CA1 in vivo, as it does during long-term potentiation in area CA1, but that the pattern of coupling of the ERK cascade to transcriptional regulators differs between the two forms of synaptic plasticity.

Key words: long-term depression; extracellular signalregulated kinase; mitogen-activated protein kinase; NMDA; cAMP response element-binding protein; Elk-1; protein phosphorylation; transcription factors
Activity-dependent changes in synaptic strength are thought to be a component of the neural substrates of learning and memory. Studies directed at uncovering the molecular mechanisms that underlie persistent changes in synaptic strength suggest that longterm potentiation (LTP) of synaptic function involves, among others, increased protein phosphorylation, whereas long-term depression (LTD) involves decreased protein phosphorylation (Bear and Malenka, 1994; Lisman, 1994; Winder and Sweatt, 2001). For instance, the activity of protein kinases and protein phosphatases was found to be altered during LTP (CharriautMarlangue et al., 1991; Fukunaga et al., 1993; Klann et al., 1993) and LTD (Thiels et al., 1998, 2000); kinases and phosphatases were found to regulate glutamate receptors after LTP- and LTDinducing stimulation, respectively (Barria et al., 1997; Lee et al., 2000) (cf. Soderling and Derkach, 2000); kinase inhibitors were found to block LTP and facilitate LTD (Frey et al., 1993; Hrabetova and Sacktor, 1996; Kameyama et al., 1998), whereas phosphatase inhibitors to block LTD and facilitate LTP (Ikegami et al., 1996; Blitzer et al., 1998; Winder et al., 1998); and genetically

Received Sept. 18, 2001; revised Dec. 13, 2001; accepted Dec. 27, 2001.

This work was supported by National Institutes of Health Grants NS36180 (E.T.) and NS34007 (E.K.). We thank Drs. J. Patrick Card and Clif W. Callaway for their invaluable help with the immunohistochemical analysis and Drs. Janice L. Hytrek, A. Christine Tabaka, James S. Piecara, and Christopher A. Teleha for the synthesis of SL327.

Correspondence should be addressed to Dr. Edda Thiels, 446 Crawford Hall, Department of Neuroscience, University of Pittsburgh, Pittsburgh, PA 15260. E-mail: thiels@bns.pitt.edu.

E. Klann's present address: Department of Molecular Physiology and Biophysics, Baylor College of Medicine, One Baylor Plaza, Houston, TX 77030.

Copyright (C) 2002 Society for Neuroscience $0270-6474 / 02 / 222054-09 \$ 15.00 / 0$ altered expression of these phospho-enzymes was found to affect LTP and/or LTD in ways consistent with the idea that changes in protein phosphorylation are part of the operations that underlie activity-dependent synaptic plasticity (Chen and Tonegawa, 1997; Winder and Sweatt, 2001).

One protein kinase demonstrated recently to play a critical role in LTP and various forms of memory is extracellular signalregulated kinase 2 (ERK2; also known as p42 mitogen-activated protein kinase) (English and Sweatt, 1997; Atkins et al., 1998; Berman et al., 1998) (cf. Thiels and Klann, 2001). Activation of ERK2 constitutes part of a signaling cascade that is triggered by receptor activation and acts on a wide range of effectors across many subcellular compartments (Grewal et al., 1999). The significance of ERK2 activation in LTP and memory, however, is thought to stem primarily from the contribution of the enzyme to the phosphorylation of the transcription factor cAMP response element-binding (CREB) protein (Xing et al., 1996). Phosphorylation of $\mathrm{CREB}$ and consequent cAMP response element (CRE)-dependent gene expression were shown to be essential for the establishment of persistent LTP and long-term memory in many systems (Alberini et al., 1995; Silva et al., 1998).

Hippocampal LTD, similar to hippocampal LTP, was found to be persistent, lasting for days in area CA1 in vivo (Doyère et al., 1996). The persistence of LTD suggests that the underlying molecular mechanisms involve altered gene expression (Kauderer and Kandel, 2000) (but see Huber et al., 2000), although the array of genes being expressed is likely to differ from that expressed during LTP. In light of the role of the ERK cascade in long-term memory and LTP, it is an open question what the status of the 
ERK cascade is during LTD. Is ERK function altered during LTD, and, if so, how does the alteration impact on transcriptional activators targeted by this kinase cascade? To answer these questions, we examined ERK phosphorylation and phosphotransferase activity and phosphorylation of CREB and of Elk-1, a regulator of serum response element (SRE)-dependent gene expression (Gille et al., 1995), during LTD in area CA1 of the adult hippocampus in vivo. Our findings indicate that activation of the ERK cascade contributes critically to the persistence of LTD and appears to promote SRE-dependent but not CRE-dependent gene expression during LTD in area CA1 of the adult hippocampus in vivo.

\section{MATERIALS AND METHODS}

Electrophysiology. Electrophysiological methods described previously (Thiels et al., 1994) were used for recordings from the hippocampus of anesthetized adult rats (Sprague Dawley, 250-350 gm; Hilltop, Scottdale, PA). All procedures were in compliance with and approved by the Institutional Animal Care and Use Committee, University of Pittsburgh. Briefly, field responses evoked by stimulation pulses $(20-250 \mu \mathrm{A}, 100$ $\mu \mathrm{sec}$ duration) delivered to the dorsal commissural pathway were recorded in either stratum pyramidale or stratum radiatum of area CA1 of the right dorsal hippocampus. Series of 10 test pulses $(0.1 \mathrm{~Hz})$ were delivered at $5 \mathrm{~min}$ intervals before and after LTD-inducing paired-pulse stimulation (PPS). PPS consisted of one train of 200 pairs of pulses, with an interstimulus interval of $25 \mathrm{msec}$ and an interpair interval of $2 \mathrm{sec}$, unless indicated otherwise, and was delivered using a stimulation intensity that produced an area CA1 population spike amplitude $\sim 60 \%$ of the maximum amplitude, as determined at the beginning of the experiment. The stimulation intensity for test pulses was set to produce a response magnitude $\sim 40 \%$ of the maximum magnitude for recordings in stratum pyramidale and $\sim 30 \%$ of the maximum magnitude for recordings in stratum radiatum, as determined at the beginning of the experiment. In some experiments, D-2-amino-5-phosphonovaleric acid (D-APV) (100 $\mu \mathrm{M}$ in the pipette, dissolved in $150 \mathrm{~mm} \mathrm{NaCl}$; Sigma, St. Louis, MO) was administered by continuous pressure ejection from a micropipette placed near the recording electrode in stratum radiatum. In other experiments, animals were injected intraperitoneally with either the ERK kinase (MEK) inhibitor SL327 [50 mg/kg, dissolved in 100\% dimethylsulfoxide (DMSO); DuPont, Wilmington, DE] or DMSO (100\%, $1 \mathrm{ml} / \mathrm{kg}$; Sigma) 60-80 min before the onset of baseline recording. We chose intraperitoneal administration for the MEK inhibitor, because intrahippocampal administration of both the inhibitor and vehicle solution caused unstable baseline responses. Recorded waveforms were amplified, filtered (0.1-10 $\mathrm{kHz}$ ), digitized $(10 \mathrm{kHz})$, and stored on computer disk for later analysis of the amplitude of the evoked area CA1 population spike or the initial slope ( $1.0 \mathrm{msec}$ after onset) of the evoked population EPSP.

For purposes of biochemical analyses, animals were killed either before or after PPS, and their right hippocampus was dissected out in the presence of cooled artificial CSF (in mM: $124 \mathrm{NaCl}, 5 \mathrm{KCl}, 1.25$ $\mathrm{NaH}_{2} \mathrm{PO}_{4}, 26 \mathrm{NaHCO}_{3}, 10$ dextrose, $1.5 \mathrm{MgCl}_{2}$, and $2.5 \mathrm{CaCl}_{2}$ ). A block of area CA1 $\left(\sim 1 \mathrm{~mm}^{3}\right)$ was excised from the dorsal and the ventral portion of the hippocampus, and each was placed in individual, coded vials on dry ice, which then were stored at $-80^{\circ} \mathrm{C}$ until biochemical analysis.

Biochemistry, tissue preparation, and Western blotting. The microdissected dorsal and ventral area CA1 tissue samples were homogenized in ice-cold buffer A (50 mM Tris-HCl, pH 7.4, 1 mM EGTA, 1 mM EDTA, $10 \mu \mathrm{M}$ benzamidine, $1 \mu \mathrm{g} / \mathrm{ml}$ aprotinin, $1 \mu \mathrm{g} / \mathrm{ml}$ leupeptin, $2 \mathrm{~mm}$ sodium pyrophosphate, $4 \mathrm{~mm} p$-nitrophenylphosphate, and $1 \mathrm{~mm}$ sodium orthovanadate) and centrifuged at $29,000 \times g$ at $4^{\circ} \mathrm{C}$ for $45 \mathrm{~min}$. The supernatant was decanted, saved, and used as soluble fraction. For preparation of nuclear extracts, the tissue samples were placed in ice-cold buffer B (10 mm HEPES-OH, pH 7.9, 10 mM KCl, 1.5 mm $\mathrm{MgCl}_{2}, 1 \mathrm{~mm}$ DTT, $1 \mathrm{~mm} \mathrm{NaF}, 1 \mathrm{~mm}$ sodium orthovanadate, $2 \mathrm{~mm}$ sodium pyrophosphate, $1 \mathrm{~mm}$ PMSF, $10 \mu \mathrm{m}$ benzamidine, $1 \mu \mathrm{g} / \mathrm{ml}$ leupeptin, $1 \mu \mathrm{g} / \mathrm{ml}$ aprotinin, and $1 \mu \mathrm{g} / \mathrm{ml}$ pepstatin) and incubated on ice for $20 \mathrm{~min}$. Cells were disrupted with a dounce homogenizer until nuclei were free of cytoskeletal attachments as detected microscopically with phase-contrast examination $(\sim 20-40$ strokes $)$ and then centrifuged at $14,000 \mathrm{rpm}$ at $4^{\circ} \mathrm{C}$ for $2 \mathrm{~min}$. The supernatant was decanted, saved, and used as cytosolic extract, and the nuclear pellet was resuspended in $30 \mu \mathrm{l}$ of buffer $\mathrm{C}$ (10
mM HEPES, pH 7.0, 450 mu NaCl, 5 mm EDTA, 0.05\% SDS, $1 \%$ Triton $\mathrm{X}-100,2 \mathrm{~mm}$ DTT, $1 \mathrm{~mm} \mathrm{NaF}, 1 \mathrm{~mm}$ sodium orthovanadate, $2 \mathrm{~mm}$ sodium pyrophosphate, $1 \mathrm{~mm}$ PMSF, $10 \mu \mathrm{M}$ benzamidine, $1 \mu \mathrm{g} / \mathrm{ml}$ leupeptin, 1 $\mu \mathrm{g} / \mathrm{ml}$ aprotinin, and $1 \mu \mathrm{g} / \mathrm{ml}$ pepstatin) and incubated for $45 \mathrm{~min}$ on ice with gentle rocking, followed by centrifugation at $14,000 \mathrm{rpm}$ at $4^{\circ} \mathrm{C}$ for $10 \mathrm{~min}$. The resulting supernatant was decanted, saved, and used as nuclear extract. To control for cytosolic contamination in the nuclear extract, we probed the nuclear fraction for aldolase $(1: 1000$; Chemicon, Temecula). To control for nuclear leakage or breakage, we probed the cytosolic extract for nuclear mitotic apparatus protein (NuMA) (1:250; Transduction Laboratories, Lexington, KY). Both of these controls yielded negative results, indicative of minimal cross-contamination.

Protein concentrations in the respective fractions were determined according to the method of Bradford (Bradford, 1976) using bovine serum albumin (BSA) as standard. Equivalent amounts of protein for each sample were resolved by $10 \%$ SDS-PAGE, blotted electrophoretically to Immobilon membranes (Millipore, Bedford, MA), blocked for 30 min with B-TTBS [50 mm Tris-HCl, pH 7.5-8.0, $150 \mathrm{~mm} \mathrm{NaCl}, 0.1 \%$ Tween 20, and 3\% BSA (phosphoERK), 5\% BSA (phosphoCREB), or $8 \%$ BSA (phosphoElk-1)], and then incubated overnight in B-TTBS with a rabbit polyclonal antibody that selectively recognizes Thr202/183- and Tyr204/185-phosphorylated, active ERK1/2 (1:5000; Promega, Madison, WI), Ser133-phosphorylated CREB (1:500; Cell Signaling Technology, Beverly, MA), or Ser383-phosphorylated Elk-1 (1:200; Cell Signaling Technology). After incubation with the primary antibody, the membrane was washed four times with TTBS buffer (50 mM Tris-HCl, pH 7.5-8.0, $150 \mathrm{~mm} \mathrm{NaCl}$, and $0.1 \%$ Tween 20 ), the blots were exposed to a donkey anti-rabbit IgG peroxidase-linked antibody (Amersham Biosciences, Piscataway, NJ) and developed using an enhanced chemiluminescence reagent (DuPont NEN, Boston, MA), and the films were analyzed densitometrically using NIH Image software. To control for protein loading, the membranes then were stripped and reprobed with either a mouse monoclonal antibody raised against either tubulin (soluble fractions; 1:400; Oncogene Sciences, Uniondale, NY) or NuMA (nuclear extracts; 1:250; Transduction Laboratories) or a rabbit polyclonal antibody raised against CREB (1:1000; Cell Signaling Technology).

ERK phosphotransferase activity assay. Tissue samples were homogenized, and protein concentration was determined as described above. Dually phosphorylated ERK was immunoprecipitated, and its ability to phosphorylate the ERK-specific substrate Elk-1 was determined using an ERK activity assay kit from Cell Signaling Technology essentially as suggested by the supplier.

Immunohistochemistry. To localize dually phosphorylated ERK within cell type and subcellular area, some animals were perfused transcardially with $30-50 \mathrm{ml}$ of physiological saline, followed by $200 \mathrm{ml}$ of $4 \%$ paraformaldehyde-lysine-periodate in $0.1 \mathrm{M}$ PBS. Brains were removed, post-fixed in $4 \%$ paraformaldehyde in PBS for $1-2 \mathrm{hr}$ at $4^{\circ} \mathrm{C}$, and then transferred to $20 \%$ sucrose in PBS, in which they were stored for 12-24 hr. Frozen brains were cut into $35 \mu \mathrm{m}$ coronal sections that were placed into cryopreservative ( $30 \%$ ethylene glycol, $30 \%$ sucrose, $1 \%$ polyvinylpyrrolidone, and $0.1 \mathrm{M}$ PBS, $\mathrm{pH} 7.4$ ) and stored at $-20^{\circ} \mathrm{C}$ until immunohistochemical analysis.

Sections at a frequency of $210 \mu \mathrm{m}$ were processed immunohistochemically by bringing them to room temperature over $30 \mathrm{~min}$ and washing them with four sequential rinses in $0.1 \mathrm{M}$ PBS containing $1 \mathrm{~mm} \mathrm{NaF}$ and $1 \mathrm{~mm}$ orthovanadate. Sections then were incubated with a rabbit polyclonal antibody that selectively recognizes Thr202/183- and Tyr204/185phosphorylated, active ERK1/2 (1:1000; Cell Signaling Technology) in $10 \mathrm{~mm}$ PBS containing $0.3 \%$ Triton X-100, $1 \mathrm{~mm} \mathrm{NaF}, 1 \mathrm{~mm}$ sodium orthovanadate, and $1 \%$ normal donkey serum at $4^{\circ} \mathrm{C}$ for $48 \mathrm{hr}$. After washing with three rinses in $10 \mathrm{~mm}$ PBS over $45 \mathrm{~min}$, sections were incubated in biotinylated donkey anti-rabbit (Jackson ImmunoResearch, West Grove, PA) for $60 \mathrm{~min}$, followed by incubation in ABC complex using reagents from the Vectastain Elite kit (Vector Laboratories, Burlingame, $\mathrm{CA}$ ) for $60 \mathrm{~min}$ at room temperature on a rotator. After washing the sections in $10 \mathrm{~mm}$ PBS, immunostaining was visualized with diaminobenzidine using the Peroxidase Substrate kit from Vector Laboratories essentially as suggested by the supplier. Sections were mounted on gelatin-coated slides, dried at room temperature, dehydrated in ethanol, cleared in xylene, and coverslipped with Cytoseal60. Sections then were examined with a Zeiss (Oberkochen, Germany) Axioplan photomicroscope equipped with differential interference optics, and images were digitized with a Dage-MTI (Michigan City, IN) video camera (MTI $3 \mathrm{CCD}$ ) and an image analysis system (Simple 32; C-Imaging Systems). 


\section{RESULTS}

\section{ERK2 activation is increased during LTD in area CA1 of the adult hippocampus in vivo}

We induced LTD in area CA1 by delivery of one train of PPS (200 pairs of impulses with a $25 \mathrm{msec}$ interstimulus interval at 0.5 $\mathrm{Hz})$ to the dorsal commissural pathway. These fibers project contralaterally from area CA3 to dorsal area CA1 but not to ventral area CA1 (Laurberg, 1979; Ishizuka et al., 1990). Figure $1 A$ shows that PPS produced a persistent depression of both the amplitude of the CA1 population spike (Fig. 1 $A a$ ) and the initial slope of the CA1 population EPSP (Fig. $1 A b$ ) evoked by test pulses delivered to the commissural fibers before and after PPS. To determine whether LTD is accompanied by a change in ERK2 activation, we removed a block of tissue from dorsal area CA1 near the recording site (experimental sample) and from ventral area CA1 from the same animal (control sample) and probed the homogenized tissue samples with an antibody that selectively recognizes ERK1/2 when phosphorylated at Thr-202/183 and Tyr-204/185, the so-called activation site of ERK1/2. Tissue blocks $\left(1 \mathrm{~mm}^{3}\right)$ were collected either immediately before PPS (baseline) or 5,15 , or $35 \mathrm{~min}$ after termination of PPS. Figure $1 B$ shows that, under basal conditions, levels of dually phosphorylated ERK2 were comparable in dorsal and ventral area CA1 (baseline: ventral vs dorsal, Student's $t$ test for matched samples, $p>0.5, n=12)$. In contrast, within 5 min after LTD-inducing stimulation, phosphoERK2 immunoreactivity was increased twofold above control levels (5 min group: control vs LTD, $p<0.05$, $n=8)$. The increase in ERK2 phosphorylation persisted for at least $15 \min (15$ min group: control vs LTD, $p<0.01, n=12$ ) but then dissipated rapidly, so that $35 \mathrm{~min}$ after LTD-inducing stimulation, phosphoERK2 immunoreactivity no longer differed from control levels (35 min group: control vs LTD, $p>0.2, n=10$ ). This profile of increased dual phosphorylation of ERK2 was confirmed in comparisons between time points using as data the level of phosphoERK2 immunoreactivity in dorsal area CA1 relative to that in ventral area CA1 determined for each animal (Wilcoxon rank sum test with Bonferroni correction; baseline vs 5 min, $p<0.05$; baseline vs $15 \mathrm{~min}, p<0.01$; baseline vs $35 \mathrm{~min}$, $p>0.5$ ). Induction of LTD with three trains of PPS (population spike amplitude $30 \mathrm{~min}$ after the last train of PPS: $14 \pm 5 \%$ of baseline, $n=4$; population EPSP slope: $74 \pm 6 \%$ of baseline, $n=$ 3) resulted in a somewhat greater increase in ERK2 phosphorylation than observed after one train of PPS (phosphoERK2 immunoreactivity 5 min after third train of PPS: $379 \pm 87 \%$ of control; control vs LTD, Student's $t$ test for matched samples, $p \leq$ $0.01, n=6)$. The increase in ERK activation after three trains of PPS, however, did not persist longer than that observed after one train of PPS (phosphoERK2 immunoreactivity $35 \mathrm{~min}$ after the third train of PPS: $104 \pm 11 \%$ of control; control vs LTD, $p>0.5$, $n=7)$.

It is feasible that the increase in ERK2 phosphorylation resulted from mere repetitive stimulation and was not linked specifically to previous induction of LTD. To test this possibility, we delivered one train of PPS in the presence of the NMDA receptor antagonist D-APV. We showed previously that this manipulation prevents the induction of LTD by PPS (Thiels et al., 1994). Consistent with our previous observations, LTD induction was blocked when PPS was delivered in the presence of D-APV (Fig. $2 A$, open circles). In parallel with the lack of LTD, delivery of PPS in the presence of D-APV was not followed by an increase in dually phosphorylated ERK2 (Fig. 2B) (PPS alone: ventral vs dorsal, Student's $t$ test for matched samples, $p<0.05, n=6$; PPS-APV: ventral vs dorsal, $p>0.5, n=5$; PPS alone vs PPS-APV, Wilcoxon rank sum test, $p<0.05$ ). These findings show that PPS alone is not sufficient to enhance ERK2 phosphorylation. We therefore conclude that induction of NMDA receptor-dependent LTD is necessary for the increase in ERK2 activation depicted in Figure 1.

Increased phosphorylation of ERK at the dual-phosphorylation site is suggestive for but not equivalent to an increase in ERK phosphotransferase activity. To test whether the LTD-associated increase in dually phosphorylated ERK2 corresponded to an increase in ERK phosphotransferase activity, we immunoprecipitated phosphorylated ERK from homogenates prepared from dorsal and ventral area CA1 removed either before or $15 \mathrm{~min}$ after PPS, added the immmunoprecipitate to an assay system containing ATP and exogenous Elk-1, and then determined phosphorylation of Elk-1 at Ser-383, a specific ERK target site, using an antibody that selectively recognizes Ser-383-phosphorylated Elk-1. Figure 3 shows that, under basal conditions, phosphorylation of the exogenous ERK substrate was comparable in assay systems containing dorsal versus ventral area CA1 homogenate (Student's $t$ test for matched samples, $p>0.2, n=6$ ). In contrast, 15 min after the induction of LTD with one train of PPS (population spike amplitude, $47 \pm 6 \%$ of baseline), phosphorylation of exogenous Elk-1 was increased twofold in assays with dorsal versus ventral area CA1 homogenate $(p<0.01, n=6)$. The difference in exogenous Elk-1 phosphorylation between baseline and 15 min after LTD induction was highly significant (Wilcoxon rank sum test, $p<0.01$ ). Analysis of ERK2 phosphorylation after LTD induction using the same tissue samples as used for the ERK activity assay revealed that phosphoERK2 immunoreactivity for dorsal area CA1 homogenate, relative to ventral area CA1 homogenate, was $243 \pm 39 \%$ (Fig. $2 B$, PPS-alone group). These findings provide strong evidence that the increase in dually phosphorylated ERK2 during LTD shown in Figure 1 is indicative for an LTD-associated increase in ERK phosphotransferase activity. Together, our findings indicate that LTD in area CA1 of the adult hippocampus in vivo involves pronounced, albeit transient, activation of the ERK cascade.

\section{ERK activation is required for LTD in area CA1 of the adult hippocampus in vivo}

Our observation that ERK activity is increased during LTD suggests the possibility that ERK is critical for the establishment of LTD. To test whether ERK activation was necessary for LTD, we treated animals with an inhibitor of MEK, the kinase directly responsible for dual phosphorylation of ERK. In agreement with previous reports (Atkins et al., 1998; Davis et al., 2000), we found in preliminary studies that intraperitoneal administration of the specific MEK inhibitor SL327 (Favata et al., 1998) caused a large decrease in ERK2 phosphorylation. PhosphoERK2 immunoreactivity for area CA1 homogenate prepared from animals treated 1-2 $\mathrm{hr}$ before tissue collection with SL327 $(50 \mathrm{mg} / \mathrm{kg}$ dissolved in $1 \mathrm{ml}$ of $100 \%$ DMSO) was $117 \pm 22$ density units compared with $482 \pm 45$ density units for area CA1 homogenate prepared from animals treated with vehicle solution (100\% DMSO; DMSO vs SL327, Student's $t$ test for independent groups, $p<0.01, n=5$ per group). Figure 4 shows that, regardless of the type of previous drug treatment, delivery of PPS produced an initial reduction of the amplitude of the evoked population spike (Fig. $4 A$ ), as well as the slope of the population EPSP (Fig. 4B). However, in animals treated with SL327, the response reduction failed to be main- 
A a

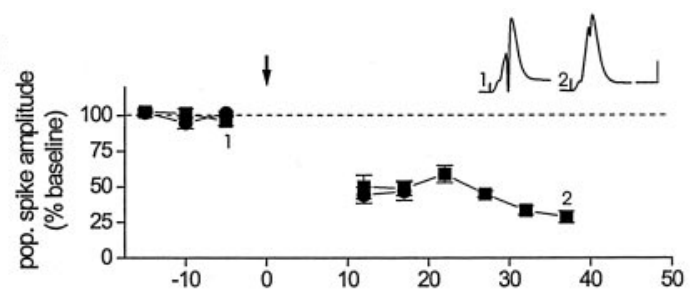

b

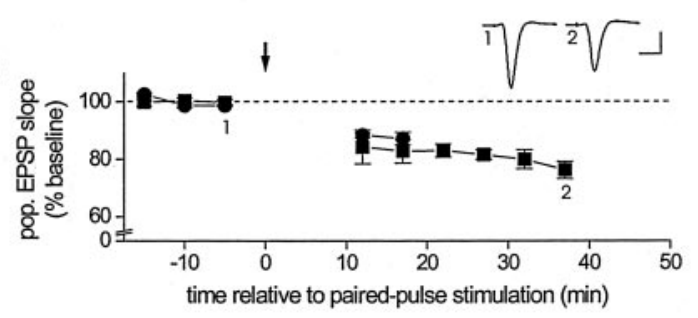

B

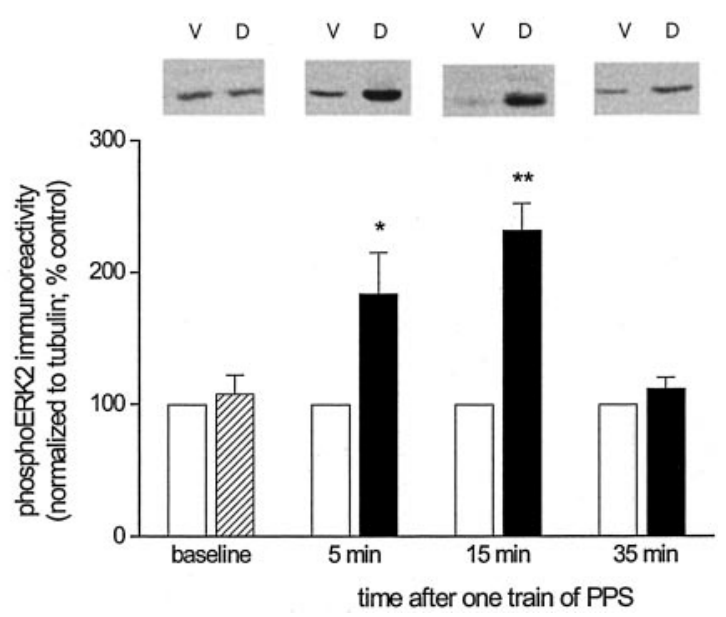

Figure 1. LTD in area CA1 of the adult hippocampus in vivo is associated with an increase in activated ERK2. $A a$, Group data (mean \pm SEM) of the amplitude of the CA1 pyramidal cell population spike evoked by stimulation of commissural fibers before and after delivery of one train of paired pulses (downward arrow) to these fibers. The data are expressed as a percentage of the average population spike amplitude before paired-pulse stimulation. Animals were killed either $15 \min ($ circles; $n=6)$ or $35 \mathrm{~min}$ (squares; $n=5$ ) after termination of paired-pulse stimulation. Inset, Average of 10 waveforms of population spikes recorded in the same animal before (1) and after (2) paired-pulse stimulation at the times indicated. Calibration: $2 \mathrm{mV}, 10 \mathrm{msec}$. $A b$, Similar group data of the initial slope of the pyramidal cell population EPSP recorded in stratum radiatum before and after paired-pulse stimulation (downward arrow). Animals were killed at the same times after termination of the stimulation train as described above (15 min, circles, $n=6 ; 35 \mathrm{~min}$, squares, $n=5$ ). Inset, Averaged waveforms of population EPSPs recorded before and after paired-pulse stimulation, as described above (calibration as above). $B$, Group data (mean \pm SEM) of dual-phosphorylated ERK2 immunoreactivity, normalized to tubulin, for ventral area CA1 homogenates (control, open bars) and dorsal area CA1 homogenates (baseline, striped bar; LTD, filled bars) derived from animals killed either $5 \mathrm{~min}$ after termination of baseline recording (baseline; $n=$ $12)$ or $5(n=8), 15(n=12)$, or $35(n=10)$ min after termination of PPS. Data are expressed as a percentage of dual-phosphorylated ERK2 immunoreactivity detected in ventral area CA1 homogenates. Dual-phosphorylated ERK2 immunoreactivity was normalized to tubulin immunoreactivity rather than total ERK immunoreactivity, because the capability of nonphospho-specific ERK antibodies to bind to ERK was found to be reduced after the induction of LTD (Norman et al., 2000). Evidence indicates that total ERK level is unaffected by the induction of LTD (Norman et al., 2000). Representative Western blots of dual-phosphorylated ERK2 for ventral $(V)$ and dorsal $(D)$ area CA1 homogenate for the time points indicated on the $x$-axis. Asterisks indicate significant difference between control and LTD samples at the indicated time points (Student's $t$ test for matched samples; * $p<0.05$; * $p<0.01$ ).
A

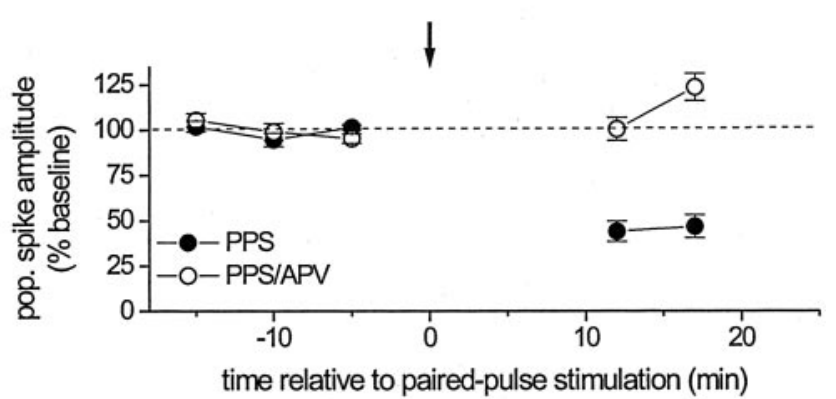

B

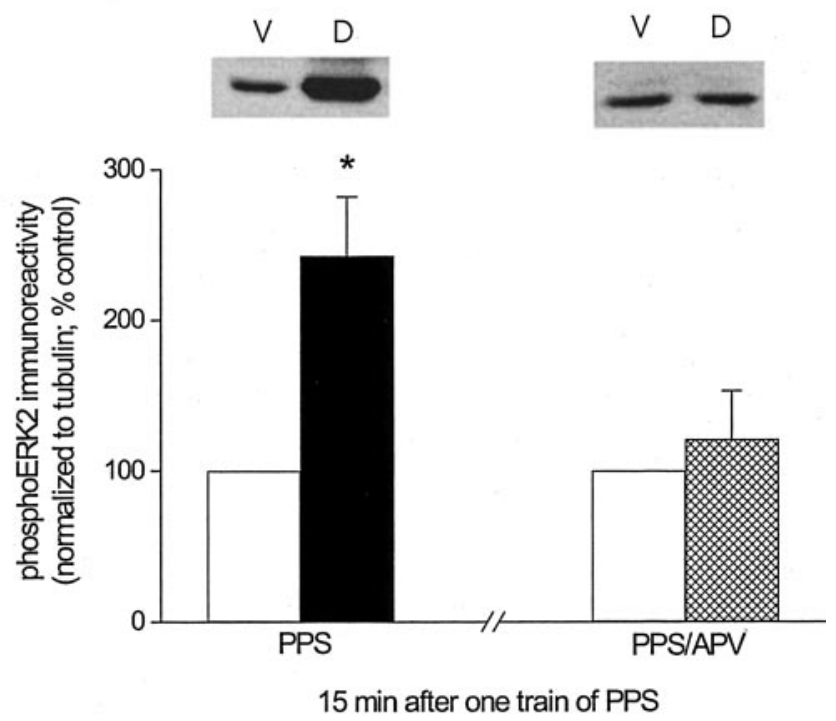

Figure 2. Blockade of NMDA receptors prevents the induction of LTD, as well as the associated increase in activated ERK2. $A$, Group data (mean \pm SEM) of the amplitude of the population spike recorded before and after paired-pulse stimulation (downward arrow) during continuous administration of the specific NMDA receptor antagonist D-APV (100 $\mu \mathrm{M}$ in the drug pipette; open circles; $n=5$ ). For purposes of comparison, the effect of paired-pulse stimulation in the absence of D-APV is depicted as well ( filled circles; $n=6$; data are taken from Fig. $1 \mathrm{Aa}$ ). $B$, Group data (mean \pm SEM) of dual-phosphorylated ERK2 immunoreactivity, normalized to tubulin, for ventral area CA1 homogenates (control, open bars) and dorsal area CA1 homogenates ( filled or cross-hatched bar) derived from animals who received paired-pulse stimulation in either the presence of D-APV (cross-hatched bar; $n=5$ ) or the absence of the drug ( filled bar; $n=6$ ) and were killed 15 min after termination of PPS. Data are expressed as a percentage of dual-phosphorylated ERK2 immunoreactivity detected in ventral area CA1 homogenates. Representative Western blots of dual-phosphorylated ERK2 for ventral $(V)$ and dorsal $(D)$ area CA1 homogenate for the two conditions indicated on the $x$-axis. Asterisk indicates significant difference between ventral and dorsal samples (Student's $t$ test for matched samples; ${ }^{*} p<0.05$ ).

tained. Within 15 min after termination of PPS, both the amplitude of the evoked population spike (Fig. 4A, filled symbols) and the slope of the evoked population EPSP (Fig. 4B, filled symbols) returned to pre-PPS baseline levels, at which they remained for the rest of the $1 \mathrm{hr}$ post-PPS recording session. In contrast, persistent LTD developed in animals treated with DMSO (Fig. 4, open symbols) (DMSO vs SL327, Student's $t$ test for independent groups, 60 min after termination of PPS, population spike amplitude: $p<0.01, n=4$ per group; population EPSP slope: $p<0.01$, $n=5$ per group).

Inhibition of MEK was reported to reduce CA1 pyramidal cell excitability (Winder et al., 1999), an effect that may have inter- 


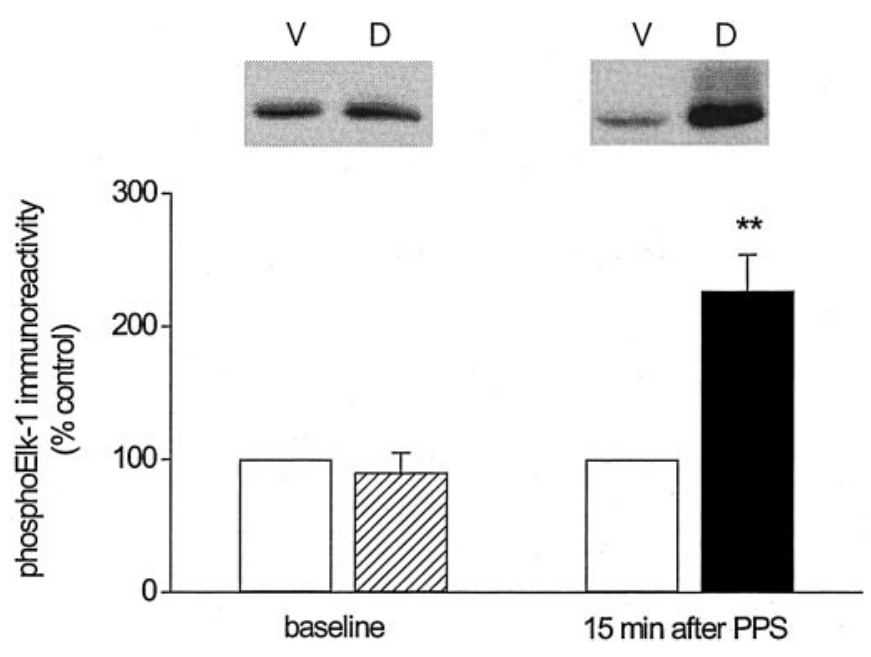

Figure 3. LTD in area CA1 of the adult hippocampus in vivo is associated with an increase in ERK phosphotransferase activity. Group data (mean \pm SEM) of Ser383-phosphorylated Elk-1 immunoreactivity in samples containing exogenously added Elk-1 and dual-phosphorylated ERK immunoprecipitated from dorsal area CA1 homogenate (baseline, striped bar; LTD, filled bar) and ventral CA1 homogenate (open bars) of animals killed either $5 \mathrm{~min}$ after baseline stimulation (baseline; $n=6$ ) or $15 \mathrm{~min}$ after termination of paired-pulse stimulation ( $15 \mathrm{~min}$ after PPS; $n=6$ ). Data are expressed as a percentage of Ser383-phosphorylated Elk-1 immunoreactivity detected in samples containing dual-phosphorylated ERK immunoprecipitated from ventral area CA1 homogenates. For additional details, see Results. Representative Western blots of Ser383-phosphorylated Elk-1 for ventral $(V)$ and dorsal $(D)$ area CA1 homogenate for the two time points indicated on the $x$-axis. Asterisks indicate significant difference between control and LTD samples (Student's $t$ test for matched samples; $* * p<0.01)$.

fered with the induction of persistent LTD. To determine whether altered pyramidal cell firing and/or total synaptic input during PPS may have contributed to the lack of persistent LTD in SL327-treated animals, we measured the amplitude of the population spike (for recordings in stratum pyramidale) and the integral of the population EPSP (for recordings in stratum radiatum) evoked by each of the pulses during PPS. We found that neither the average population spike amplitude (DMSO, $1.2 \pm 0.4$ vs $\mathrm{SL} 327,1.7 \pm 0.7 \mathrm{mV} ; n=4$ per group) nor the average population EPSP integral (DMSO, $50 \pm 10$ vs SL327, $40 \pm 12 \mathrm{mV} / \mathrm{msec} ; n=$ 5 per group) during PPS differed significantly between the two drug conditions (Student's $t$ tests for independent groups, both $p$ values $\geq 0.5$ ). Because induction of LTD was shown to require inhibition of pyramidal cell firing to the second pulse of each pair during PPS (Thiels et al., 1994), we compared the two drug conditions with respect to this variable as well. We found that, in both groups, the average amplitude of the population spike evoked by the second pulse of each pair was inhibited greatly across much of the train (DMSO, $2 \pm 2 \%$ of baseline amplitude vs SL327, $1 \pm 1 \%$ of baseline amplitude; Student's $t$ test for independent groups, $p>0.3$ ). Together, these findings suggest that the difference in LTD persistence between SL327-treated and DMSO-treated animals depicted in Figure 4 cannot readily be accounted for in terms of differential cell firing, total synaptic input, or paired-pulse inhibition during the induction train. We therefore favor the conclusion that the MEK inhibitor interfered with persistent LTD because ERK activation is a necessary step in the sequelae that underlie the maintenance and/or expression of LTD.
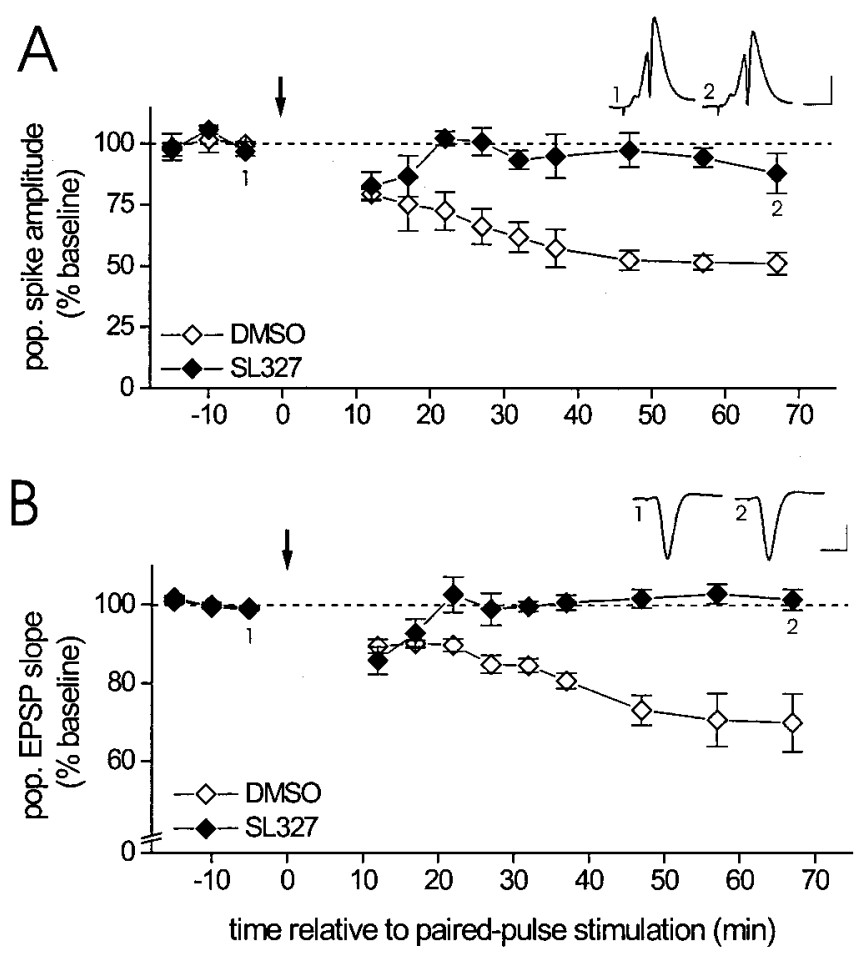

Figure 4. Inhibition of the ERK kinase MEK prevents the expression of LTD in area CA1 of the adult hippocampus in vivo. A, Group data (mean \pm SEM) of the amplitude of the CA1 pyramidal cell population spike evoked by stimulation of commissural fibers before and after delivery of one train of paired-pulse stimulation (downward arrow) after animals were treated with either the MEK inhibitor SL327 (50 mg $/ \mathrm{kg}$, i.p.; filled diamonds; $n=4)$ or vehicle solution (100\% DMSO, $1 \mathrm{ml} / \mathrm{kg}$, i.p.; open diamonds; $n=4) 1-2 \mathrm{hr}$ before paired-pulse stimulation. For additional details, see Results. Inset, Average of 10 waveforms of population spikes recorded in the same SL327-treated animal before (1) and after (2) paired-pulse stimulation at the times indicated. Calibration: 2 $\mathrm{mV}, 10 \mathrm{msec} . B$, Similar group data of the initial slope of the pyramidal cell population EPSP recorded in stratum radiatum before and after paired-pulse stimulation (downward arrow) after treatment with either SL327 ( filled diamonds; $n=5$ ) or DMSO (open diamonds; $n=5$ ). Inset, Averaged waveforms of population EPSPs recorded in a SL327-treated animal before and after paired-pulse stimulation, as described above (calibration as above).

\section{Elk-1 phosphorylation but not CREB phosphorylation is increased during LTD in area CA1 of the adult hippocampus in vivo}

Effectors of the ERK cascade that have received much attention in the context of persistent changes in synaptic function are constitutively expressed transcriptional regulators (Impey et al., 1998; Davis et al., 2000). As a first step toward determining whether ERK activated during LTD might impact on nuclear targets, we sought to localize dually phosphorylated ERK at the cellular and subcellular level after the induction of LTD. To that end, we performed immunohistochemistry using a dual-phosphospecific ERK1/2 antibody on sections of dorsal area CA1 from animals perfused either before or $10-15 \mathrm{~min}$ after PPS. Figure $5 A$ shows that staining for dually phosphorylated ERK was very low under basal conditions (baseline; left panels). In contrast, after PPS, pronounced immunostaining was present in clusters of pyramidal cells, with labeling being detectable in apical dendritic regions, as well as cell bodies of these cells (LTD; right panels). These findings of enhanced phosphoERK immunostaining in the soma of pyramidal cells suggest that LTD may involve an increase 
A
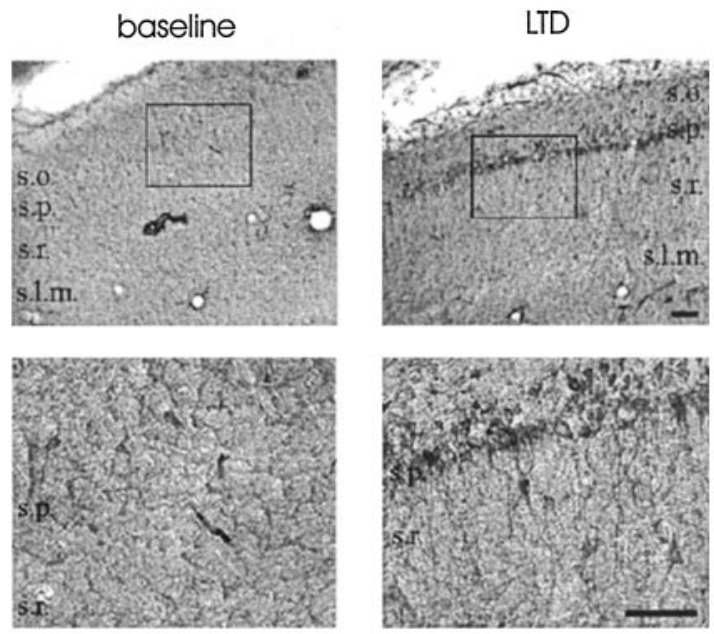

B
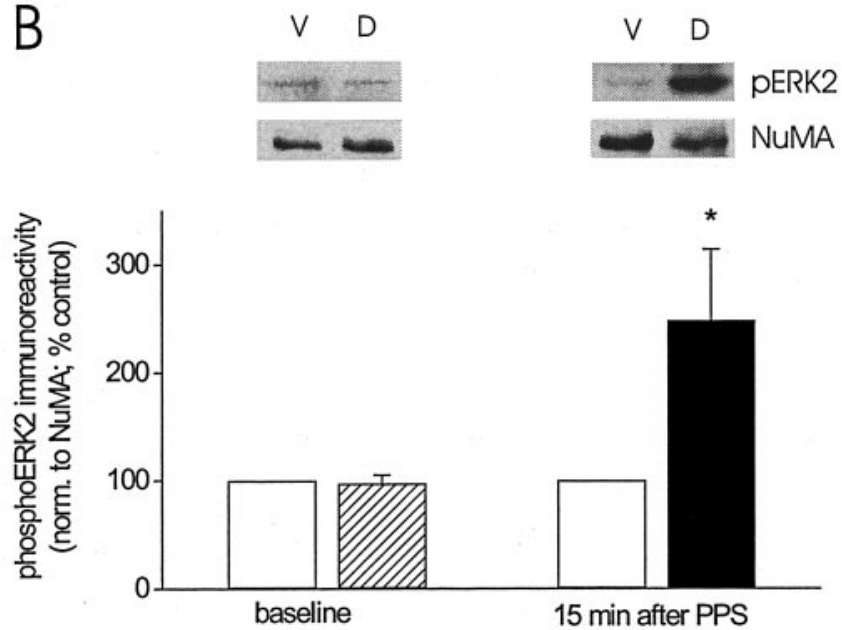

Figure 5. LTD in area CA1 of the adult hippocampus in vivo is associated with an increase in activated ERK in CA1 pyramidal cell dendrites and somata, including nuclei. $A$, Dual-phosphorylated ERK1/2 staining in sections of area CA1 from an animal that received baseline stimulation only (baseline) and an animal that received baseline and paired-pulse stimulation (LTD). Tissue sections from the two animals were processed simultaneously. Bottom panels show the boxed section marked in the respective top panels at a higher magnification. Scale bar, $100 \mu \mathrm{m}$. Similar patterns of staining and differences in staining intensity after baseline stimulation versus LTD-inducing stimulation were observed in other pairs of animals $(n=4)$. $B$, Group data (mean \pm SEM) of dual-phosphorylated ERK2 immunoreactivity, normalized to NuMA, for nuclear extracts prepared from ventral area CA1 (control, open bars) and dorsal area CA1 (baseline, striped bar; LTD, filled bar) collected from animals killed either $5 \mathrm{~min}$ after termination of baseline recording (baseline; $n=4$ ) or $15 \mathrm{~min}$ after termination of paired-pulse stimulation ( 15 min after PPS; $n=5$ ). Data are expressed as a percentage of dual-phosphorylated ERK2 immunoreactivity detected in nuclear extracts from ventral area CA1. Representative Western blots of dual-phosphorylated ERK2 ( $p E R K 2)$ and of NuMA for nuclear extracts from ventral $(V)$ and dorsal $(D)$ area CA1 for the two time points indicated on the $x$-axis. Asterisk indicates significant difference between control and LTD samples (Student's $t$ test for matched samples; * $p<0.05)$

in activated ERK in pyramidal cell nuclei. To test this possibility directly, we prepared nuclear extracts from tissue of dorsal and ventral area CA1 collected either before or $15 \mathrm{~min}$ after PPS and probed the extracts for dually phosphorylated ERK1/2. Figure $5 B$ shows that levels of dually phosphorylated ERK2 were compara- ble in nuclear extract from dorsal and ventral area CA1 before PPS (baseline: ventral vs dorsal, Student's $t$ test for matched samples, $p>0.5, n=4)$. After PPS, however, phosphoERK2 immunoreactivity in nuclear extracts was significantly higher than control levels ( 15 min group: $p<0.05, n=5$; baseline vs $15 \mathrm{~min}$ after PPS: Wilcoxon rank sum test, $p=0.06$ ). Together, these observations indicate increased nuclear levels of activated ERK2 during LTD and, hence, raise the possibility that nuclear targets of the ERK cascade are affected during LTD.

To examine the phosphorylation status of two nuclear effectors of the ERK cascade, namely, CREB and Elk-1, we prepared nuclear extracts from tissue of dorsal and ventral area CA1 as described above and probed the extracts for either Ser133phosphorylated CREB or Ser383-phosphorylated Elk-1. Figure $6 \mathrm{~A}$ shows that no difference was found in phosphoCREB immunoreactivity between dorsal and ventral area CA1 nuclear extracts before PPS (baseline: $p>0.5, n=5$ ). To our surprise, phosphorylation of CREB was decreased significantly from control levels after PPS (15 min group: $p<0.05, n=6$; baseline vs 15 min after PPS: Wilcoxon rank sum test, $p<0.05$ ). Total CREB immunoreactivity was comparable between dorsal and ventral area CA1 before and after PPS (CREB immunoreactivity for dorsal area CA1, as a percentage of that for ventral area CA1; baseline: $92 \pm 7 \%$, ventral vs dorsal, Student's $t$ test for matched samples, $p>0.2 ; 15$ min group: $106 \pm 14 \%$, ventral vs dorsal, $p>$ 0.5 ), which indicates that the decrease in CREB phosphorylation after PPS was not attributable to a loss in total CREB. The effect of PPS on Elk-1 phosphorylation is depicted in Figure 6B. Consistent with the LTD-associated increase in ERK activation, phosphorylation of Elk-1 was increased markedly above control levels after PPS (15 min group: ventral vs dorsal, Student's $t$ test for matched samples, $p<0.01, n=8$ ). No difference in phosphoElk-1 immunoreactivity was observed between experimental and control samples before PPS (baseline: ventral vs dorsal, $p>0.5, n=4$; baseline vs 15 min after PPS: Wilcoxon rank sum test, $p<0.01$ ). To test whether the increase in Elk-1 phosphorylation was mediated by ERK activation, we determined Elk-1 phosphorylation in nuclear extracts prepared from animals treated with SL327 (50 mg/kg) 1-2 hr before PPS. We found that the previously observed increase in Elk-1 phosphorylation was abolished completely in the presence of the MEK inhibitor (phosphoElk-1 immunoreactivity for nuclear extracts from dorsal area CA1, relative to that for nuclear extracts from ventral area CA1, 15 min after PPS: $98 \pm 7 \%$, ventral vs dorsal, Student's $t$ test for matched samples, $p>0.5, n=5$ ). Collectively, these findings indicate that Elk-1 phosphorylation is increased during LTD in the adult hippocampus in vivo and that this increase in Elk-1 phosphorylation is likely to be a functional consequence of enhanced activation of the ERK cascade during LTD.

\section{DISCUSSION}

Growing evidence implicates activation of the ERK cascade as a critical event in the establishment of long-term memories and persistent LTP (Orban et al., 1999; Thiels and Klann, 2001). Similar to hippocampal LTP, hippocampal LTD has been shown to persist for days (Doyère et al., 1996), and LTD-like changes in synaptic function have been proposed to be integral to the acquisition and storage of memories (Hopfield et al., 1983; Kohonen, 1984; Willshaw and Dayan, 1990). Indeed, acquisition of new spatial information was linked recently to facilitated induction of LTD (Manahan-Vaughan and Braunewell, 1999). Moreover, hippocampal LTD, similar to persistent hippocampal LTP, appears 
A
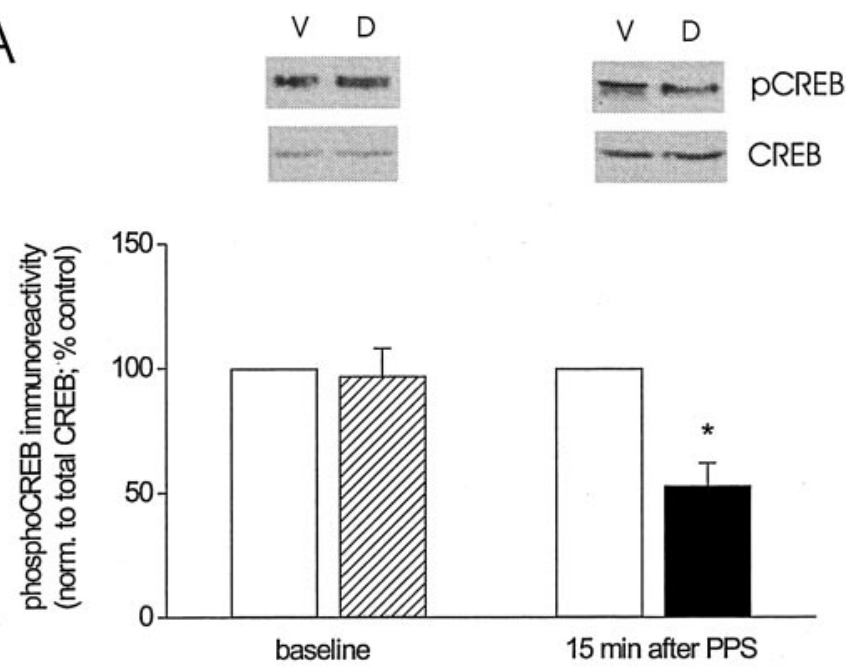

B

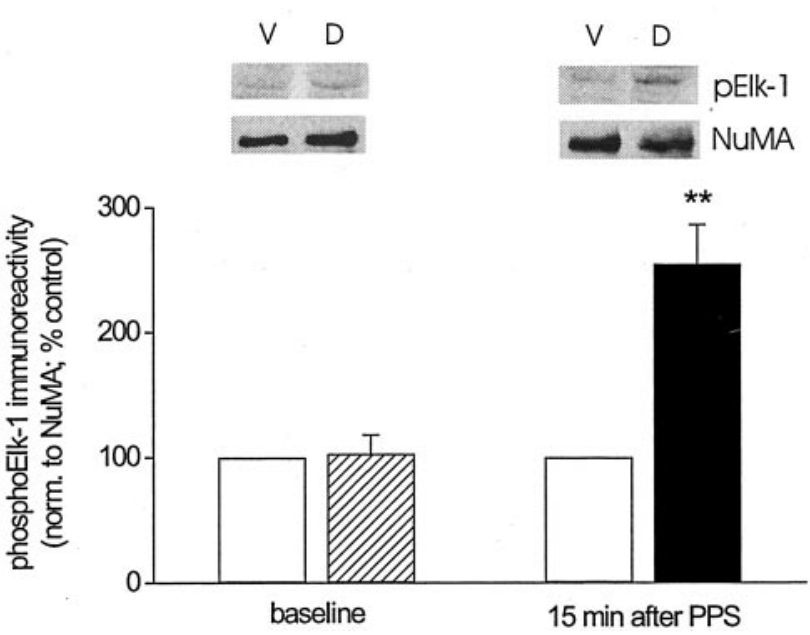

Figure 6. LTD in area CA1 of the adult hippocampus in vivo is associated with a decrease in nuclear CREB phosphorylation but an increase in nuclear Elk-1 phosphorylation. $A$, Group data (mean \pm SEM) of Ser133phosphorylated CREB immunoreactivity, normalized to total CREB, for nuclear extracts prepared from ventral area CA1 (control, open bars) and dorsal area CA1 (baseline, striped bar; LTD, filled bars) collected from animals killed either $5 \mathrm{~min}$ after termination of baseline recording (baseline; $n=5$ ) or 15 min after termination of paired-pulse stimulation (15 min after PPS; $n=6$ ). Data are expressed as a percentage of Ser133phosphorylated CREB immunoreactivity detected in nuclear extracts from ventral area CA1. Representative Western blots of Ser133phosphorylated CREB ( $p C R E B)$ and total CREB for nuclear extracts from ventral $(V)$ and dorsal $(D)$ area CA1 for the two time points indicated on the $x$-axis (Student's $t$ test for matched samples; * $p<0.05$ ). $B$, Similar group data for Ser383-phosphorylated Elk-1 immunoreactivity, normalized to NuMA, for nuclear extracts prepared from ventral area CA1 (control, open bars) and dorsal area CA1 (baseline, striped bar; LTD, filled bars) collected from animals killed either 5 min after termination of baseline recording (baseline; $n=4$ ) or $15 \mathrm{~min}$ after termination of paired-pulse stimulation (15 min after PPS; $n=7)$. Representative Western blots of Ser383-phosphorylated Elk-1 ( $p E l k-1)$ and NuMA for nuclear extracts from ventral $(V)$ and dorsal $(D)$ area CA1 for the two time points indicated on the $x$-axis. PhosphoElk-1 immunoreactivity was normalized to NuMA immunoreactivity because of an inconsistent detectability of the total Elk-1 signal. In those cases in which total Elk-1 could be detected, no difference was noted between normalization to Elk-1 and to NuMA. Asterisks indicate significant difference between control and LTD samples (Student's $t$ test for matched samples; ${ }^{* *} p<$ $0.01)$. to require de novo transcription (Kauderer and Kandel, 2000), which suggests that, in both forms of synaptic plasticity, signaling cascades are recruited that transduce synaptic signals into transcriptional signals. The ERK cascade is thought to contribute to such a signal transduction process during LTP. We here showed that ERK may play a similar role during LTD.

First, we demonstrated that NMDA receptor-dependent LTD induced in area CA1 of the adult hippocampus in vivo involves a robust increase in ERK2 phosphorylation and ERK1/2 phosphotransferase activity. These findings are consistent with previous immunocytochemical findings showing that a variety of stimulation frequencies and intensities, including low-stimulation frequencies, applied to the Schäffer collateral/commissural fibers produce ERK activation in CA1 pyramidal cells maintained in vitro (Dudek and Fields, 2001). In light of findings of increased ERK2 activation during both NMDA receptor-dependent LTP in area CA1 in vitro (English and Sweatt, 1996) and NMDA receptor-dependent LTD in area CA1 in vivo (present findings), it becomes an interesting question whether the magnitude, kinetics, and/or subcellular localization of ERK activation during LTD differ from those during LTP at the same synapse in the same preparation. Relevant to this issue are findings that, during LTD, ERK1/2 becomes modified outside the dual-phosphorylation site through a mechanism mediated by okadaic acid-sensitive protein phosphatases, such as protein phosphatases 1 and 2A (Norman et al., 2000). Based on these findings and observations of increased protein phosphatase 1 and 2A activity during LTD in area CA1 in vivo (Thiels et al., 1998), one might expect that the extent of ERK activation during LTD is attenuated somewhat relative to that during LTP at the commissural-CA1 pyramidal cell synapse in vivo.

Second, we demonstrated that ERK activation is necessary for the persistent maintenance and expression of NMDA receptordependent LTD in area CA1 of the adult hippocampus in vivo. When ERK activation was prevented through inhibition of MEK, PPS produced only a transient reduction in synaptic transmission. ERK activation was found to be necessary for high-frequency stimulation-induced LTP but not for theta burst stimulationinduced LTP (Winder et al., 1999). Whether the dependence of LTD on the ERK cascade also varies as a function of stimulation protocol (and, hence, pattern and route of calcium influx and types of receptors activated) remains to be determined. In this respect, it is interesting to note that, in contrast to the present findings, no change in ERK1/2 phosphorylation was observed during metabotropic glutamate receptor-dependent LTD induced in area CA1 of hippocampal slices from young animals (Bolshakov et al., 2000). This difference in engagement of the ERK cascade between NMDA receptor-dependent LTD and metabotropic glutamate receptor-dependent LTD adds to the growing evidence that these two forms of LTD involve distinct signaling cascades.

Third, we demonstrated that the subcellular compartments experiencing an increase in activated ERK during LTD include CA1 pyramidal cell nuclei. Monitoring the subcellular localization and movement of total ERK during LTD is difficult because of the aforementioned LTD-associated modification of the enzyme (Norman et al., 2000). We therefore were unable to determine whether the increase in activated ERK in the nucleus resulted from translocation of the activated enzyme from the cytosol to the nucleus. Nuclear translocation of activated ERK was shown to occur during LTP (Impey et al., 1998) and, hence, may also underlie the increase in nuclear ERK signal observed 
here. Once in the nucleus, activated ERK can target a variety of nuclear phosphoproteins, including regulators of transcription.

Fourth, we demonstrated that Elk-1 phosphorylation becomes increased during LTD and that this increase requires activation of ERK. ERK-dependent phosphorylation of Elk-1 has been observed previously after associative learning in cortex (Berman et al., 1998) and after LTP induction in the dentate gyrus (Davis et al., 2000). Elk-1 is a member of the ternary complex factor transcription factors and a direct target of ERK (Gille et al., 1995). In complex with serum response factor, phosphorylated Elk-1 can bind to SREs and regulate SRE-dependent gene expression (Wasylyk et al., 1998). SREs are in the promoter region of several immediate early genes (IEGs), including of IEGs transcriptionally induced during activity-dependent synaptic plasticity (Abraham et al., 1993; Worley et al., 1993). Thus, our observations of an ERK-dependent increase in Elk-1 phosphorylation suggest that expression of SRE-containing IEGs may be induced after the induction of LTD and that activation of the ERK cascade in response to LTD-inducing synaptic activation plays a critical role in regulating IEG induction.

In addition to Elk-1, CREB is a nuclear target of the ERK cascade whose phosphorylation state is increased after various patterns of synaptic activation (Deisseroth et al., 1996). In contrast to the positive coupling between the ERK cascade and CREB phosphorylation observed during LTP (Impey et al., 1998; Davis et al., 2000), we found CREB phosphorylation to be decreased during LTD. The decrease in CREB phosphorylation despite increased ERK activation may be attributable to dephosphorylation of ribosomal S6 protein kinase (RSK), the intermediary kinase between ERK and CREB (Xing et al., 1996), or dephosphorylation of CREB itself. Both RSK and CREB are substrates for protein phosphatases, including those shown to be activated during LTD (Mulkey et al., 1993; Bito et al., 1996; Thiels et al., 1998). In any event, our findings of decreased CREB phosphorylation during LTD suggest that CRE-driven gene expression may be depressed after the induction of LTD. Interestingly, many promoter regions that contain a ternary complex factor binding site also contain a CREB protein binding site (Wasylyk et al., 1998). The present pattern of findings of increased Elk-1 phosphorylation and decreased CREB phosphorylation therefore suggests the intriguing possibility that the decrease in CREB phosphorylation may attenuate Elk-1-mediated IEG induction during LTD. Future studies aimed at examining the impact of decreased, as well as increased, phosphorylation of transcriptional regulators on gene expression during LTD will shed light on this issue.

\section{REFERENCES}

Abraham WC, Demmer J, Richardson LC, Williams JM, Lawlor PA, Mason SE, Tate WP, Dragunow M (1993) Correlations between early gene induction and the persistence of LTP. Neuroscience 56:717-727.

Alberini CM, Ghirardi M, Huang Y-Y, Nguyen PV, Kandel ER (1995) A molecular switch for the consolidation of long-term memory: cAMPinducible gene expression. Ann NY Acad Sci 758:261-286.

Atkins CM, Selcher JC, Petraitis JJ, Trzaskos JM, Sweatt JD (1998) The MAPK cascade is required for mammalian associative learning. Nat Neurosci 1:602-609.

Barria A, Muller D, Derkach V, Griffith LC, Soderling TR (1997) Regulatory phosphorylation of AMPA-type glutamate receptors by CaMKII during long-term potentiation. Science 276:2042-2045.

Bear MF, Malenka RC (1994) Synaptic plasticity: LTP and LTD. Curr Opin Neurobiol 4:389-399.

Berman DE, Hazvi S, Rosenblum K, Seger R, Dudai Y (1998) Specific and differential activation of mitogen-activated protein kinase cascades by unfamiliar taste in the insular cortex of the behaving rat. J Neurosci 18:10037-10044.

Bito H, Deisseroth K, Tsien RW (1996) CREB phosphorylation and dephosphorylation: a $\mathrm{Ca}^{2+}$ - and stimulus duration-dependent switch for hippocampal gene expression. Cell 87:1203-1214.

Blitzer RD, Connor JH, Brown GP, Wong T, Shenolikar S, Iyengar R, Landau EM (1998) Gating of CaMKII by cAMP-regulated protein phosphatase activity during LTP. Science 280:1940-1943.

Bolshakov VY, Carboni L, Cobb MH, Siegelbaum SA, Belardetti F (2000) Dual MAP kinase pathways mediate opposing forms of longterm plasticity at CA3-CA1 synapses. Nat Neurosci 3:1107-1112.

Bradford MM (1976) A rapid and sensitive method for the quantitation of microgram quantities of protein utilizing the principle of protein dye binding. Anal Biochem 72:248-254.

Charriaut-Marlangue C, Otani S, Creuzt C, Ben-Ari Y, Loeb J (1991) Rapid activation of hippocampal casein kinase II during long-term potentiation. Proc Natl Acad Sci USA 88:10232-10236.

Chen C, Tonegawa S (1997) Molecular genetic analysis of synaptic plasticity, activity-dependent neural development, learning, and memory in the mammalian brain. Annu Rev Neurosci 20:157-184.

Davis S, Vanhoutte P, Pagès C, Caboche J, Laroche S (2000) The MAPK/ERK cascade targets both Elk-1, cAMP response elementbinding protein to control long-term potentiation-dependent gene expression in the dentate gyrus in vivo. J Neurosci 20:4563-4572.

Deisseroth K, Bito H, Tsien RW (1996) Signaling from synapse to nucleus: postsynaptic CREB phosphorylation during multiple forms of hippocampal synaptic plasticity. Neuron 16:89-101.

Doyère V, Errington ML, Laroche S, Bliss TVP (1996) Low-frequency trains of paired stimuli induce long-term depression in area CA1 but not in the dentate gyrus of the intact rat. Hippocampus 6:52-57.

Dudek SM, Fields RD (2001) Mitogen-activated protein kinase/extracellular signal-regulated kinase activation in somatodendritic compartments: Roles of action potentials, frequency, and mode of calcium entry. J Neurosci 21:RC122(1-5).

English JD, Sweatt JD (1996) Activation of p42 mitogen-activated protein kinase in hippocampal long term potentiation. J Biol Chem 271:24329-24332.

English JD, Sweatt JD (1997) A requirement for the mitogen-activated protein kinase cascade in hippocampal long-term potentiation. J Biol Chem 272:19103-19106.

Favata M, Horiuchi KY, Manos EJ, Daulerio AJ, Stradley DA, Feeser WS, Van Dyk DE, Pitts WJ, Earl RA, Hobbs F, Copeland RA, Magolda RL, Scherle PA, Trzaskos JM (1998) Identification of a novel inhibitor of mitogen-activated protein kinase kinase. J Biol Chem 273:18623-18632.

Frey U, Huang Y-Y, Kandel ER (1993) Effects of cAMP simulate a late stage of LTP in hippocampal CA1 neurons. Science 260:1661-1664.

Fukunaga K, Stoppini L, Miyamoto E, Muller D (1993) Long-term potentiation is associated with increased activity of $\mathrm{Ca}^{2+} /$ calmodulindependent protein kinase II. J Biol Chem 268:7863-7867.

Gille H, Kortenjamm M, Thomae O, Moomaw C, Slaughter C, Cobb MH, Shaw PE (1995) ERK phosphorylation potentiates Elk-1-mediated ternary complex formation and transactivation. EMBO J 14:951-962.

Grewal SS, York RD, Stork PJS (1999) Extracellular-signal-regulated kinase signalling in neurons. Curr Opin Neurobiol 9:544-553.

Hopfield JJ, Feinstein DI, Palmer RG (1983) "Unlearning" has a stabilizing effect in collective memories. Nature 304:158-159.

Hrabetova S, Sacktor TC (1996) Bidirectional regulation of protein kinase $\mathrm{M} \zeta$ in the maintenance of long-term potentiation and long-term depression. J Neurosci 16:5324-5333.

Huber KM, Kayser MS, Bear MF (2000) Role for rapid dendritic protein synthesis in hippocampal mGluR-dependent long-term depression. Science 288:1254-1256.

Ikegami S, Kato A, Kudo Y, Kuno T, Ozawa F, Inokuchi K (1996) A facilitatory effect on the induction of long-term potentiation in vivo by chronic administration of antisense oligodeoxynucleotides against catalytic subunits of calcineurin. Mol Brain Res 41:183-191.

Impey S, Obrietan K, Wong ST, Poser S, Yano S, Wayman G, Deloulme JC, Chan G, Storm DR (1998) Cross talk between ERK and PKA is required for $\mathrm{Ca}^{2+}$ stimulation of CREB-dependent transcription and ERK nuclear translocation. Neuron 21:869-883.

Ishizuka N, Weber J, Amaral DG (1990) Organization of intrahippocampal projections originating from $\mathrm{CA} 3$ pyramidal cells in the rat. J Comp Neurol 295:580-623.

Kameyama K, Lee H-K, Bear MF, Huganir RL (1998) Involvement of postsynaptic protein kinase A substrate in the expression of homosynaptic long-term depression. Neuron 21:1163-1175.

Kauderer BS, Kandel ER (2000) Capture of a protein synthesisdependent component of long-term depression. Proc Natl Acad Sci USA 97:13342-13347.

Klann E, Chen S-J, Sweatt JD (1993) Mechanism of protein kinase C activation during the induction and maintenance of long-term potentiation probed using a selective peptide substrate. Proc Natl Acad Sci USA 90:8337-8341.

Kohonen T (1984) Self-organization and associative memory. Berlin: Springer.

Laurberg S (1979) Commissural and intrinsic connections of the rat hippocampus. J Comp Neurol 184:685-708. 
Lee H, Barbarosie M, Kameyama K, Bear MF, Huganir RL (2000) Regulation of distinct AMPA receptor phosphorylation sites during bidirectional synaptic plasticity. Nature 405:955-959.

Lisman J (1994) The CaMKII hypothesis for the storage of synaptic memory. Trends Neurosci 17:406-412.

Manahan-Vaughan D, Braunewell K-H (1999) Novelty acquisition is associated with induction of hippocampal long-term depression. Proc Natl Acad Sci USA 96:8739-8744.

Mulkey RM, Herron CE, Malenka RC (1993) Essential role for protein phosphatases in hippocampal long-term depression. Science 261:1051-1055.

Norman ED, Thiels E, Barrionuevo G, Klann E (2000) Long-term depression in the hippocampus in vivo is associated with protein phosphatase-dependent alterations in extracellular signal-regulated kinase. J Neurochem 74:192-198.

Orban PC, Chapman PF, Brambilla R (1999) Is the Ras-MAPK signalling pathway necessary for long-term memory formation? Trends Neurosci 22:38-44.

Silva AJ, Kogan JH, Frankland PW, Kida S (1998) CREB and memory. Annu Rev Neurosci 21:127-148.

Soderling TR, Derkach VA (2000) Postsynaptic protein phosphorylation and LTP. Trends Neurosci 23:75-80.

Thiels E, Klann E (2001) Extracellular signal-regulated kinase, synaptic plasticity, and memory. Rev Neurosci 12:327-345.

Thiels E, Barrionuevo G, Berger TW (1994) Excitatory stimulation during postsynaptic inhibition induces long-term depression in hippocampus in vivo. J Neurophysiol 72:3009-3016.

Thiels E, Norman ED, Barrionuevo G, Klann E (1998) Transient and persistent increases in protein phosphatase activity during long-term depression in the adult hippocampus in vivo. Neuroscience 86:10231029.

Thiels E, Kanterewicz BI, Knapp LT, Barrionuevo G, Klann E (2000) Protein phosphatase-mediated regulation of protein kinase $\mathrm{C}$ during long-term depression in the adult hippocampus in vivo. J Neurosci 20:7199-7207.

Wasylyk B, Hagman J, Gutierrez-Hartmann A (1998) Ets transcription factors: nuclear effectors of the Ras-MAP-kinase signaling pathway. Trends Biochem Sci 23:213-216.

Willshaw D, Dayan P (1990) Optimal plasticity from matrix memories; what goes up must come down. Neural Comput 2:85-93.

Winder DG, Sweatt JD (2001) Roles of serine/threonine phosphatases in hippocampal synaptic plasticity. Nat Rev 2:1-14.

Winder DG, Mansuy IM, Osman M, Moallem TM, Kandel ER (1998) Genetic and pharmacological evidence for a novel, intermediate phase of long-term potentiation suppressed by calcineurin. Cell 92:25-37.

Winder DG, Martin KC, Muzzio IA, Rohrer D, Chruscinski A, Kobilka B, Kandel ER (1999) ERK plays a regulatory role in the induction of LTP by theta frequency stimulation and its modulation by $\beta$-adrenergic receptors. Neuron 24:715-726.

Worley PF, Bhat RV, Baraban JM, Erickson CA, McNaughton BL, Barnes CA (1993) Thresholds for synaptic activation of transcription factors in hippocampus: correlation with long-term enhancement. J Neurosci 13:4776-4786.

Xing J, Ginty DD, Greenberg ME (1996) Coupling of the RAS-MAPK pathway to gene activation by RSK2, a growth factor-regulated CREB kinase. Science 273:959-963. 